\title{
HARMONIC MAPS AND COSYMPLECTIC MANIFOLDS
}

\author{
E. BOECKX and C. GHERGHE
}

(Received 15 February 2002; revised 19 January 2003)

Communicated by $\mathrm{K}$. Wysocki

\begin{abstract}
We study the harmonicity of maps to or from cosymplectic manifolds by relating them to maps to or from Kähler spaces.
\end{abstract}

2000 Mathematics subject classification: primary 53C25, 53C43, 58E20.

Keywords and phrases: cosymplectic manifolds, harmonic maps, energy, $(\varphi, J)$-holomorphic maps, $\varphi$-pluriharmonic maps.

\section{Introduction}

The theory of harmonic maps between Riemannian manifolds has taken an enormous flight since its conception [3-6]. Combining both global and local aspects and borrowing both from Riemannian geometry and from analysis, the theory has developed in many diverse branches. In particular, there is now a whole battery of deep and interesting results about harmonic maps to or from complex manifolds and Kähler spaces. These even-dimensional spaces can be described using complex coordinates, and hence one can use the methods and results from complex function theory.

Within contact geometry, there are several classes of manifolds that can be considered as odd-dimensional analogs of Kähler spaces, the most important ones being Sasakian and cosymplectic spaces. Even though many of the concepts of Kähler geometry have counterparts in contact geometry, the theory of harmonic maps to or from contact manifolds is only in its initial stages $[8,10]$. One reason seems to be the absence of something like complex coordinates for these manifolds.

In this paper, we develop a theory of harmonic maps and cosymplectic manifolds analogous to the one in the Kähler context. The idea is not to mimic the proofs

(C) 2004 Australian Mathematical Society $1446-7887 / 04 \$ A 2.00+0.00$ 
for Kähler manifolds (which may be nearly impossible when these use complex coordinates) but instead to use the results for Kähler manifolds to prove similar results for cosymplectic manifolds. In order for this scheme to work, we must be able to go back and forth between cosymplectic and Kähler manifolds and between corresponding mappings. This turns out to be surprisingly easy, but at the same time constitutes a very powerful tool.

The paper is organized as follows. After recalling the necessary facts about harmonic maps between general Riemannian manifolds, we motivate why we consider cosymplectic manifolds (rather than, say, Sasakian manifolds). Then we describe how to construct a Kähler space from a cosymplectic manifold and how to 'lift' mappings accordingly. These lifts behave very well both with respect to harmonicity and with respect to the cosymplectic and Kähler structures. In Section 5 and Section 6, we put our construction to work to prove various results about harmonic maps and cosymplectic manifolds, analogs of known results for Kähler spaces.

During the preparation of this manuscript, the authors visited each other's universities in the context of an agreement between the Royal Flemish Academy of Belgium for Sciences and Arts and the Romanian Academy. They want to express their gratitude to both Academies for their financial support.

\section{Harmonic maps on Riemannian manifolds}

In this section we recall some well-known general facts concerning harmonic maps.

Let $\left(M^{m}, g\right)$ and $\left(N^{n}, h\right)$ be two Riemannian manifolds and $F:(M, g) \rightarrow(N, h)$ a smooth map. The energy density of $F$ is the smooth function $e(F): M \rightarrow[0, \infty)$ given by

$$
e(F)_{p}=\frac{1}{2}\left\|d F_{p}\right\|^{2}=\frac{1}{2} \operatorname{Tr}\left(F^{*} h\right)(p)=\frac{1}{2} \sum_{i=1}^{m} h\left(d F\left(e_{i}\right), d F\left(e_{i}\right)\right)
$$

for $p \in M$ and any orthonormal basis $\left\{e_{1}, \ldots, e_{m}\right\}$ of $T_{p} M$. If $M$ is a compact Riemannian manifold, then the energy $E(F)$ of $F$ is the integral of its energy density

$$
E(F)=\int_{M} e(F) \mu_{M},
$$

where $\mu_{M}$ is the volume measure associated with the metric $g$ on $M$. A map $F:(M, g) \rightarrow(N, h)$ is said to be harmonic if it is a cricital point of the energy functional $E$ on the set of all maps between $(M, g)$ and $(N, h)$.

In order to describe the critical point condition for the functional $E$, we look at the differential $d F$. It is a section of the bundle $T^{*} M \otimes F^{-1} T N \rightarrow M$. This bundle has a connection $\nabla^{\prime}$ induced from the Levi Civita connection $\nabla^{M}$ on $T M$ and the pull-back 
connection $\nabla^{F}$ on $F^{-1} T N$. Applying this connection to $d F$, one obtains the second fundamental form $\alpha_{F} \in \Gamma\left(\odot^{2} T^{*} M \otimes F^{-1} T N\right)$. Explicitly,

$$
\alpha_{F}(X, Y)=\left(\nabla^{\prime} d F\right)(X, Y)=\nabla_{X}^{F}(d F(Y))-d F\left(\nabla_{X}^{M} Y\right),
$$

for vector fields $X, Y \in \Gamma(T M)$. A map $F$ for which $\alpha_{F}$ vanishes identically is called totally geodesic. We will also need the second fundamental form of the composition of two smooth maps $F$ and $G$. This is given by the formula (see, for example, [4])

$$
\alpha_{G \circ F}=d G \circ \alpha_{F}+\alpha_{G}(d F, d F)
$$

The section $\tau(F) \in \Gamma\left(F^{-1} T N\right)$, defined by $\tau(F)=\operatorname{Tr}_{g} \alpha_{F}$ is called the tension field of $F$. A smooth map $F$ on a compact Riemannian manifold $M$ is harmonic precisely when $\tau(F) \equiv 0$ on $M$ [3]. More generally, we say that a map $F$ on $M$ is harmonic if it satisfies the critical condition $\tau(F)=0$, regardless of the compactness of $M$.

Now, let $(M, g)$ be a compact Riemannian manifold and $F:(M, g) \rightarrow(N, h)$ a harmonic map. We take a smooth variation $F_{s, t}$ with parameters $s, t \in(-\varepsilon,+\varepsilon)$ and with $F_{0,0}=F$. The corresponding variation fields are denoted by $V$ and $W$. The Hessian $H_{F}$ of a harmonic map $F$ is defined by

$$
H_{F}(V, W)=\left.\frac{\partial^{2} E\left(F_{s, t}\right)}{\partial s \partial t}\right|_{(s, t)=(0,0)} .
$$

The second variation formula of $E$ is $[13,18]$

$$
H_{F}(V, W)=\int_{M} h\left(J_{F}(V), W\right) \mu_{M},
$$

where $J_{F}$ is a second-order selfadjoint elliptic differential operator acting on the space of variation vector fields along $F$ (which can be identified with $\Gamma\left(F^{-1}(T N)\right)$ ), and is defined by

$$
J_{F}(V):=-\sum_{i=1}^{m}\left(\nabla_{e_{i}}^{F} \nabla_{e_{i}}^{F}-\nabla_{\nabla_{i}^{\mu} e_{i}}^{F}\right) V-\sum_{i=1}^{m} R^{N}\left(V, d F\left(e_{i}\right)\right) d F\left(e_{i}\right)
$$

for any $V \in \Gamma\left(F^{-1}(T N)\right)$ and any local orthonormal frame $\left\{e_{1}, \ldots, e_{m}\right\}$ on $M$. Here, $R^{N}$ is the curvature tensor of $(N, h)$.

The index of a harmonic map $F$ is defined as the dimension of the largest subspace of $\Gamma\left(F^{-1}(T N)\right)$ on which the Hessian $H_{F}$ is negative definite. A harmonic map $\mathrm{F}$ is said to be stable if its index is zero and otherwise is said to be unstable. 


\section{Cosymplectic manifolds}

Let $M$ be a smooth manifold of dimension $2 n+1$. We recall that an almost contact structure on $M$ is a triple $(\xi, \eta, \varphi)$, where $\xi$ is a vector field, $\eta$ is a one-form and $\varphi$ is a tensor field of type $(1,1)$ which satisfy [1]

$$
\varphi^{2}=-\mathrm{Id}+\eta \otimes \xi \quad \text { and } \quad \eta(\xi)=1,
$$

where Id is the identity endomorphism on $T M$. Then we have $\varphi \xi=0$ and $\eta \circ \varphi=0$. Furthermore, if $g$ is an associated Riemannian metric on $M$, that is, a metric which satisfies

$$
g(\varphi X, \varphi Y)=g(X, Y)-\eta(X) \eta(Y),
$$

then we say that $(\xi, \eta, \varphi, g)$ is an almost contact metric structure. A manifold equipped with such a structure is an almost contact metric manifold. The existence of an almost contact structure on $M$ is equivalent to the existence of a reduction of the structural group to $U(n) \times 1$. The fundamental 2-form $\Phi$ of an almost contact metric manifold $M$ is defined by $\Phi(X, Y)=g(X, \varphi Y)$ for $X, Y \in \Gamma(T M)$.

An almost contact manifold $(M, \xi, \eta, \varphi)$ is said to be normal if the almost complex structure $J$ on $M \times \mathbb{R}$ given by

$$
J\left(X, a \frac{d}{d t}\right)=\left(\varphi X-a \xi, \eta(X) \frac{d}{d t}\right),
$$

where $a$ is a $C^{\infty}$ function on $M \times \mathbb{R}$, is integrable, which is equivalent to the condition $N_{\varphi}+2 d \eta \otimes \xi=0$, where $N_{\varphi}$ denotes the Nijenhuis tensor of $\varphi$.

Now, let $(\xi, \eta, \varphi, g)$ be an almost contact metric structure on $M$. We define an almost Hermitian structure $(J, h)$ on $M \times \mathbb{R}$, where $J$ is the above almost complex structure and $h$ is the Hermitian metric defined by

$$
h\left(\left(X, a \frac{d}{d t}\right),\left(Y, b \frac{d}{d t}\right)\right)=g(X, Y)+a b .
$$

An almost contact metric structure $(\xi, \eta, \varphi, g)$ is said to be trans-Sasakian if $M \times$ $\mathbb{R}$ endowed with the almost Hermitian structure $(J, h)$ belongs to the class $\omega_{4}$ in the classification of Gray and Hervella [9]. Equivalently, an almost contact metric manifold $(M, \xi, \eta, \varphi, g)$ of dimension $2 n+1$ is trans-Sasakian if and only if [16]

$$
\left(\nabla_{X}^{M} \varphi\right)(Y)=\alpha\{g(X, Y) \xi-\eta(Y) X\}+\beta\{g(\varphi X, Y) \xi-\eta(Y) \varphi X\}
$$

where $\alpha=\delta \Phi(\xi) / 2 n$ and $\beta=-\delta \eta / 2 n$. 
An almost contact metric structure $(\xi, \eta, \varphi, g)$ is said to be $\mathscr{C}_{5}$ if it is trans-Sasakian with $\alpha=0$; Kenmotsu if it is $\mathscr{C}_{5}$ with $\beta=1 ; \mathscr{C}_{6}$ if it is trans-Sasakian with $\beta=0$; Sasakian if it is $\mathscr{C}_{6}$ with $\alpha=1$; cosymplectic if it is trans-Sasakian with $\alpha=\beta=0$.

In this article, we are interested in a theory of harmonic maps on almost contact metric spaces. As these are the odd-dimensional analogues of almost Hermitian manifolds, it is instructive to look at harmonic maps on such spaces. If $(M, J, h)$ is a Kähler manifold and $N$ is a Riemannian manifold, then a smooth map $F: M \rightarrow N$ is called pluriharmonic if its second fundamental form $\alpha_{F}$ satisfies the condition

$$
\alpha_{F}(X, Y)+\alpha_{F}(J X, J Y)=0
$$

for any $X, Y \in \Gamma(T M)$. Clearly, any pluriharmonic map is a harmonic map [15].

In [10], an analogous concept is considered for the class of almost contact metric manifolds. If $(M, \xi, \eta, \varphi, g)$ is an almost contact metric manifold and $N$ is a Riemannian manifold, then a smooth map $F: M \rightarrow N$ is called $\varphi$-pluriharmonic if

$$
\alpha_{F}(X, Y)+\alpha_{F}(\varphi X, \varphi Y)=0
$$

for any $X, Y \in \Gamma(T M)$. In particular, $\alpha_{F}(X, \xi)=0$ for any tangent vector $X$. It is not difficult to show that $\varphi$-pluriharmonicity implies harmonicity.

Secondly, one can look at structure-preserving mappings between almost Hermitian and almost contact metric manifolds, as analogues of the well-known holomorphic mappings in complex geometry. There are three different situations:

1. A smooth map $F: M \rightarrow N$ from an almost contact metric manifold $(M, \xi, \eta$, $\varphi, g)$ to an almost Hermitian manifold $(N, J, h)$ is $(\varphi, J)$-holomorphic if $d F \circ \varphi=$ $J \circ d F$. Note that $d F(\xi)=0$ for such a map.

2. A smooth map $F: N \rightarrow M$ from an almost Hermitian manifold $(N, J, h)$ to an almost contact metric manifold $(M, \xi, \eta, \varphi, g)$ is $(J, \varphi)$-holomorphic if $d F \circ J=$ $\varphi \circ d F$. Now, Im $d F \perp \xi$.

3. A smooth map $F: M_{1} \rightarrow M_{2}$ between almost contact metric manifolds $\left(M_{i}, \xi_{i}\right.$, $\left.\eta_{i}, \varphi_{i}, g_{i}\right), i=1,2$, is $\varphi$-holomorphic if $d F \circ \varphi_{1}=\varphi_{2} \circ d F$. In particular, $d F\left(\xi_{1}^{\perp}\right) \subset \xi_{2}^{\perp}$ and $d F\left(\xi_{1}\right) \sim \xi_{2}$.

When $d F$ intertwines the structures up to a minus sign, we speak about $(\varphi, J)$-antiholomorphic, $(J, \varphi)$-anti-holomorphic and $\varphi$-anti-holomorphic mappings.

PROPOSITION 3.1. Any $(\varphi, J)$-holomorphic mapping $F$ from a cosymplectic manifold $M$ to a Kähler manifold $N$ is $\varphi$-pluriharmonic and thus a harmonic map.

Proof. Using the formula $d F \circ \varphi=J \circ d F$ we easily find

$$
J\left(\alpha_{F}(X, Y)\right)+\left(\nabla_{d F(X)}^{N} J\right)(d F(Y))=d F\left(\left(\nabla_{X}^{M} \varphi\right) Y\right)+\alpha_{F}(X, \varphi Y)
$$


for any $(\varphi, J)$-holomorphic map from an almost contact metric manifold to an almost Hermitian manifold. (A similar formula holds for holomorphic, $(J, \varphi)$-holomorphic and $\varphi$-holomorphic maps, see [8].)

Now, if $M$ is a cosymplectic manifold and $N$ is a Kähler manifold, then $\nabla^{M} \varphi=0$ and $\nabla^{N} J=0$, and we have $J\left(\alpha_{F}(X, Y)\right)=\alpha_{F}(X, \varphi Y)$ for any $X, Y \in \Gamma(T M)$. Replacing $Y$ by $\varphi Y$, we obtain $J\left(\alpha_{F}(X, \varphi Y)\right)=-\alpha_{F}(X, Y)$. Using the symmetry of $\alpha_{F}$, we have $J\left(\alpha_{F}(\varphi X, \varphi Y)\right)=-\alpha_{F}(\varphi X, Y)=-J\left(\alpha_{F}(X, Y)\right)$ and hence $\alpha_{F}(X, Y)+\alpha_{F}(\varphi X, \varphi Y)=0$ for any $X, Y \in \Gamma(T M)$. So $F$ is $\varphi$-pluriharmonic and thus harmonic.

It is known that the Hopf fibration $S^{2 m+1} \rightarrow \mathbb{C} P^{m}$ is a harmonic map from a Sasakian manifold onto a Kähler manifold. On the other hand, it easy to show that the Hopf fibration is not a $\varphi$-pluriharmonic map. So it is natural to ask under which conditions a $(\varphi, J)$-holomorphic map is $\varphi$-pluriharmonic. Within the class of trans-Sasakian manifolds, we have a full answer.

THEOREM 3.2. Let $(M, \varphi, \xi, \eta, g)$ be a trans-Sasakian manifold, $(N, J, h)$ a Kähler manifold and $F: M \rightarrow N$ a $(\varphi, J)$-holomorphic submersion. If $F$ is $\varphi$-pluriharmonic, then $M$ is a cosymplectic manifold.

Proof. We recall that $d F(\xi)=0$ for a $(\varphi, J)$-holomorphic map. As $M$ is transSasakian and $N$ is Kähler, we have from (2)

$$
d F\left(\left(\nabla_{X}^{M} \varphi\right) Y\right)=-\eta(Y)\{\alpha d F(X)+\beta d F(\varphi X)\} .
$$

Using (3) we obtain

$$
J\left(\alpha_{F}(X, Y)\right)=-\eta(Y)\{\alpha d F(X)+\beta d F(\varphi X)\}+\alpha_{F}(X, \varphi Y)
$$

for any $X, Y \in \Gamma(T M)$. On the other hand, as $F$ is a $\varphi$-pluriharmonic map, we have $\alpha_{F}(X, \xi)=0$ for any $X \in \Gamma(T M)$. So, taking $Y=\xi$ in the above relation, we obtain

$$
\alpha d F(X)+\beta d F(\varphi X)=0
$$

for any $X \in \Gamma(T M)$.

Now, if we replace $X$ by $\varphi X$, we obtain $\alpha d F(\varphi X)-\beta d F(X)=0$ for any $X \in \Gamma(T M)$. From the last two relations it follows that $\left(\alpha^{2}+\beta^{2}\right) d F(X)=0$ for any $X \in \Gamma(T M)$. As $F$ is a submersion, this implies that $\alpha=\beta=0$, that is, $M$ is a cosymplectic manifold.

COROLlARY 3.3. There are no $(\varphi, J)$-holomorphic $\varphi$-pluriharmonic maps from a Sasakian (or Kenmotsu) manifold into a Kähler manifold.

The above results indicate that cosymplectic manifolds are the first candidates for a nice theory of harmonic maps in the context of contact geometry. 


\section{Products of cosymplectic manifolds}

We have already mentioned in the introduction that we want to use the results about harmonic maps on Kähler manifolds to deduce similar ones for harmonic maps on cosymplectic manifolds. To do this, we build a Kähler manifold starting from a cosymplectic manifold.

Let $\left(M_{1}, \xi_{1}, \eta_{1}, \varphi_{1}, g_{1}\right)$ and $\left(M_{2}, \xi_{2}, \eta_{2}, \varphi_{2}, g_{2}\right)$ be two almost contact metric structures. On $M_{1} \times M_{2}$ we define the $(1,1)$-tensor field $J^{\times}$by

$$
J^{\times}\left(X_{1}, X_{2}\right)=\left(\varphi_{1} X_{1}-\eta_{2}\left(X_{2}\right) \xi_{1}, \varphi_{2} X_{2}+\eta_{1}\left(X_{1}\right) \xi_{2}\right)
$$

for $X=\left(X_{1}, X_{2}\right) \in T\left(M_{1} \times M_{2}\right)$. One easily checks that $J^{\times} \circ J^{\times}=-$Id, hence $J^{\times}$is an almost complex structure. Moreover, for the product metric $g^{\times}=g_{1}+g_{2}$ on $M_{1} \times M_{2}$, it holds $g^{\times}\left(J^{\times} X, J^{\times} Y\right)=g^{\times}(X, Y)$ for all $X, Y \in T\left(M_{1} \times M_{2}\right)$. Hence, $\left(M_{1} \times M_{2}, J^{\times}, g^{\times}\right)$is an almost Hermitian manifold. The almost complex structure $J^{\times}$ was first defined in [14]; the almost Hermitian structure $\left(J^{\times}, g^{\times}\right)$on $M_{1} \times M_{2}$ was studied in [2]. In particular, it was proved there

PROPOSITION 4.1. The almost Hermitian structure $\left(J^{\times}, g^{\times}\right)$on $M_{1} \times M_{2}$ is almost Kähler if and only if the almost contact metric structures $\left(\xi_{1}, \eta_{1}, \varphi_{1}, g_{1}\right)$ and $\left(\xi_{2}, \eta_{2}, \varphi_{2}, g_{2}\right)$ are both almost cosymplectic (that is, $d \eta_{1}=d \eta_{2}=d \Phi_{1}=d \Phi_{2}=0$ ). Moreover, it is a Kähler structure if and only if both almost contact metric structures are cosymplectic.

It is worth noting that the Calabi-Eckmann and the Hopf manifolds are special cases of the above construction $[19,22]$.

In the sequel, when starting from an (almost) cosymplectic manifold $(M, \xi, \eta$, $\varphi, g$ ), we will associate to it the (almost) Kähler manifold obtained from the above product structure where we take $M_{1}=M_{2}=M$. We denote this manifold and its structure by $\left(M^{\times}, J^{\times}, g^{\times}\right)$.

The next step is to 'lift' maps from or into $M$ to maps from or into the product manifold $M^{\times}$. For now, let us forget about the cosymplectic structure on $M$ and only concentrate on metrical aspects. Since, as a Riemannian space, $\left(M^{\times}, g^{\times}\right)$is simply a Riemannian product, the following is valid for arbitrary Riemannian manifolds, and even for more general products. We need three types of lifts in the sequel, depending whether we switch to the product manifold on both the source and the target manifold, or only on one of the two.

1. Consider a map $f_{1}:\left(M_{1}, g_{1}\right) \rightarrow\left(M_{2}, g_{2}\right)$. We define an associated map $F_{1}$ by

$$
F_{1}:\left(M_{1}^{\times}, g_{1}^{\times}\right) \rightarrow\left(M_{2}^{\times}, g_{2}^{\times}\right):(x, y) \mapsto\left(f_{1}(x), f_{1}(y)\right) .
$$


2. For a map $f_{2}:(M, g) \rightarrow(N, h)$, define the lift

$$
F_{2}:\left(M^{\times}, g^{\times}\right) \rightarrow(N, h):(x, y) \rightarrow f_{2}(x) .
$$

Note that $F_{2}=f_{2} \circ \pi_{1}$, where $\pi_{1}$ is the natural projection of $M \times M$ on the first factor.

3. Finally, we lift a map $f_{3}:(N, h) \rightarrow(M, g)$ to

$$
F_{3}:(N, h) \rightarrow\left(M^{\times}, g^{\times}\right): x \mapsto\left(f_{3}(x), a\right)
$$

for an arbitrary fixed $a \in M$. Note that $F_{3}=i_{1} \circ f_{3}$, where $i_{1}: M \rightarrow M \times M$ : $x \mapsto(x, a)$ is the natural embedding of $M$ into $M \times M$ as first factor.

PROPOSITION 4.2. With the maps $f_{1}, f_{2}, f_{3}$ and their lifts $F_{1}, F_{2}, F_{3}$ as above, it holds that $f_{i}$ is a harmonic map if and only if $F_{i}$ is a harmonic map. Further, if the source manifold is compact, we have

$$
E\left(F_{1}\right)=2 \operatorname{vol}\left(M_{1}\right) E\left(f_{1}\right), \quad E\left(F_{2}\right)=\operatorname{vol}(M) E\left(f_{2}\right), \quad E\left(F_{3}\right)=E\left(f_{3}\right) .
$$

PROOF. The proof goes by simple computation. Consider first the second fundamental form of $F_{1}$. It is given in terms of $f_{1}$ by

$$
\alpha_{F_{1}}=\left(\alpha_{f_{1}}\left(d \pi_{1}, d \pi_{1}\right), \alpha_{f_{1}}\left(d \pi_{2}, d \pi_{2}\right)\right) .
$$

As the projections $\pi_{1}, \pi_{2}: M_{1} \times M_{1} \rightarrow M_{1}$ are Riemannian submersions, we can take traces on both sides to obtain $\tau\left(F_{1}\right)(x, y)=\left(\tau\left(f_{1}\right)(x), \tau\left(f_{1}\right)(y)\right)$. Hence, $F_{1}$ is harmonic if and only if $f_{1}$ is.

For $F_{2}=f_{2} \circ \pi_{1}$, we use formula (1) to get

$$
\alpha_{F_{2}}=d f_{2} \circ \alpha_{\pi_{1}}+\alpha_{f_{2}}\left(d \pi_{1}, d \pi_{1}\right)=\alpha_{f_{2}}\left(d \pi_{1}, d \pi_{1}\right)
$$

as $\pi_{1}$ is totally geodesic. Taking traces, we have $\tau\left(F_{2}\right)=\tau\left(f_{2}\right)$ and $F_{2}$ is harmonic if and only if $f_{2}$ is.

For the lift $F_{3}=i_{1} \circ f_{3}$, we get in a similar way

$$
\alpha_{F_{3}}=d i_{1} \circ \alpha_{f_{3}}+\alpha_{i_{1}}\left(d f_{3}, d f_{3}\right)=d i_{1} \circ \alpha_{f_{3}}
$$

as $i_{1}$ is totally geodesic. Taking traces, we obtain $\tau\left(F_{3}\right)=d i_{1}\left(\tau\left(f_{3}\right)\right)$. Since $d i_{1}$ is one-to-one, $\tau\left(F_{3}\right)=0$ if and only if $\tau\left(f_{3}\right)=0$.

The assertions about the energy can be checked easily. As an example, we have for $F_{1}$

$$
\begin{aligned}
E\left(F_{1}\right) & =\int_{M_{1} \times M_{1}}\left|d F_{1}\right|^{2}(x, y) \mu_{M_{1}}(x) \wedge \mu_{M_{1}}(y) \\
& =\int_{M_{1} \times M_{1}}\left(\left|d f_{1}\right|^{2}(x)+\left|d f_{1}\right|^{2}(y)\right) \mu_{M_{1}}(x) \wedge \mu_{M_{1}}(y)=2 \operatorname{vol}\left(M_{1}\right) E\left(f_{1}\right) .
\end{aligned}
$$

The other equalities are proved similarly. 
Moreover, the three lifts preserve stability of harmonic mappings.

PROPOSITION 4.3. Take harmonic maps $f_{1}, f_{2}, f_{3}$ on compact source manifolds and let $F_{1}, F_{2}, F_{3}$ be the corresponding lifted mappings. Then $f_{i}$ is a stable harmonic map if and only if $F_{i}$ is.

Proof. We only give the detailed calculations for the lift $F_{2}$. The other cases are similar.

Suppose that $f_{2}:(M, g) \rightarrow(N, h)$ is harmonic and $M$ is compact. Then, as we have just seen, also $F_{2}:\left(M^{\times}, g^{\times}\right) \rightarrow(N, h):(x, y) \mapsto f_{2}(x)$ is harmonic. Consider a vector field $V$ along $F_{2}$. In a local orthonormal frame $\left\{H_{1}, \ldots, H_{n}\right\}$ on $N$, we can decompose $V$ as $V=\sum_{k=1}^{n} v_{k}(x, y) H_{k}$.

Further, let $\left\{E_{1}, \ldots, E_{m}\right\}$ and $\left\{E_{1}^{\prime}, \ldots, E_{m}^{\prime}\right\}$ be local orthonormal frames on $M$ around the points $a \in M$ and $b \in M$, respectively. Then, with slight abuse of notation, $\left\{E_{1}, \ldots, E_{m}, E_{1}^{\prime}, \ldots, E_{m}^{\prime}\right\}$ is a local orthonormal frame on $M^{\times}$around $(a, b)$. Then we have

$$
\nabla_{E_{i}}^{F_{2}} V=\sum E_{i}\left(v_{k}\right) H_{k}+\sum v_{k} \nabla_{E_{i}}^{F_{2}} H_{k}=\sum E_{i}\left(v_{k}\right) H_{k}+\sum v_{k} \nabla_{E_{i}}^{f_{2}} H_{k}
$$

or, more precisely,

$$
\begin{aligned}
\left(\nabla_{E_{i}}^{F_{2}} V\right)(a, b) & =\left.\left.\sum E_{i}\left(v_{k}(x, b)\right)\right|_{x=a} H_{k}\right|_{f_{2}(a)}+\left.\sum v_{k}(a, b)\left(\nabla_{E_{i}}^{f_{2}} H_{k}\right)\right|_{f_{2}(a)} \\
& =\left(\nabla_{E_{i}}^{f_{2}} V(\cdot, b)\right)(a) .
\end{aligned}
$$

On the other hand, as $d F_{2}\left(E_{i}^{\prime}\right)=0,\left(\nabla_{E_{i}^{\prime}}^{F_{2}} V\right)(a, b)=\left.\left.\sum E_{i}^{\prime}(v(a, y))\right|_{y=b} H_{k}\right|_{f_{2}(a)}$. Hence, we obtain for the Hessian of the energy functional

$$
\begin{aligned}
H_{F_{2}}(V, V) & \\
= & \int_{M \times M}\left(\left|\nabla^{F_{2}} V\right|^{2}(x, y)\right. \\
& -\sum_{i} h_{F_{2}(x, y)}\left(R^{N}\left(V(x, y), d F_{2}\left(E_{i}\right)\right) d F_{2}\left(E_{i}\right), V(x, y)\right) \\
& \left.-\sum_{i} h_{F_{2}(x, y)}\left(R^{N}\left(V(x, y), d F_{2}\left(E_{i}^{\prime}\right)\right) d F_{2}\left(E_{i}^{\prime}\right), V(x, y)\right)\right) \mu_{M}(x) \wedge \mu_{M}(y) \\
= & \int_{M \times M}\left(\left|\nabla^{f_{2}} V(\cdot, y)\right|^{2}(x)+\sum_{i}\left|\nabla_{E_{i}^{\prime}}^{F_{2}} V\right|^{2}(x, y)\right. \\
& \left.-\sum_{i} h_{f_{2}(x)}\left(R^{N}\left(V(\cdot, y), d f_{2}\left(E_{i}\right)\right) d f_{2}\left(E_{i}\right), V(\cdot, y)\right)(x)\right) \mu_{M}(x) \wedge \mu_{M}(y)
\end{aligned}
$$




$$
=\int_{M} H_{f_{2}}(V(\cdot, y), V(\cdot, y)) \mu_{M}(y)+\int_{M \times M} \sum_{i}\left|\nabla_{E_{i}^{\prime}}^{F_{2}} V\right|^{2} \mu_{M}(x) \wedge \mu_{M}(y) .
$$

Since the second term is always non-negative, it follows that stability of $f_{2}$ implies stability of $F_{2}$.

Conversely, consider a variation vector field $\bar{V}$ along $f_{2}$. For the vector field $V(x, y)=\bar{V}(x)$ along $F_{2}$, it holds $\nabla_{E_{i}^{\prime}}^{F_{2}} V=0$, and the formula for the Hessian above reduces to $H_{F_{2}}(V, V)=\operatorname{vol}(M) H_{f_{2}}(\bar{V}, \bar{V})$. Hence, stability of $F_{2}$ implies stability of $f_{2}$ too.

We now specialize the three types of lifts above, taking also the cosymplectic structure on $M$ into account. Consider a map $f_{1}: M_{1} \rightarrow M_{2}$ between two cosymplectic manifolds and its lift $F_{1}: M_{1}^{\times} \rightarrow M_{2}^{\times}:(x, y) \mapsto\left(f_{1}(x), f_{1}(y)\right)$ between Kähler manifolds.

PROPOSITION 4.4. $F_{1}$ is a holomorphic map between Kähler manifolds if and only if $f_{1}$ is a $\varphi$-holomorphic map between cosymplectic manifolds.

PROOF. Suppose first that $f_{1}$ is $\varphi$-holomorphic, that is, $d f_{1} \circ \varphi_{1}=\varphi_{2} \circ d f_{1}$. As noted before, this implies $d f_{1}\left(\xi_{1}^{\perp}\right) \subset \xi_{2}^{\perp}$ and $d f_{1}\left(\xi_{1}\right)=a \xi_{2}$ for some function $a$ on $M_{1}$. In particular, $\eta_{2}(d f(X))=a \eta_{1}(X)$. Then, for $X, Y \in T M_{1}$, we have

$$
\begin{aligned}
\left(d F_{1} \circ J_{1}^{\times}\right)(X, Y) & =d F_{1}\left(\varphi_{1} X-\eta_{1}(Y) \xi_{1}, \varphi_{1} Y+\eta_{1}(X) \xi_{1}\right) \\
& =\left(d f_{1}\left(\varphi_{1} X\right)-a \eta_{1}(Y) \xi_{2}, d f_{1}\left(\varphi_{1} Y\right)+a \eta_{1}(X) \xi_{2}\right) \\
& =\left(\varphi_{2} d f_{1}(X)-\eta_{2}\left(d f_{1}(Y)\right) \xi_{2}, \varphi_{2} d f_{1}(Y)+\eta_{2}\left(d f_{1}(X)\right) \xi_{2}\right) \\
& =J_{2}^{\times}\left(d f_{1}(X), d f_{1}(Y)\right)=\left(J_{2}^{\times} \circ d F_{1}\right)(X, Y) .
\end{aligned}
$$

Hence, $F_{1}$ is holomorphic.

Conversely, suppose that $F_{1}$ is holomorphic. Then, for $X \in T M_{1}$, we have

$$
\left(d F_{1} \circ J_{1}^{\times}\right)(X, 0)=d F_{1}\left(\varphi_{1} X, \eta_{1}(X) \xi_{1}\right)=\left(d f_{1}\left(\varphi_{1} X\right), \eta_{1}(X) d f_{1}\left(\xi_{1}\right)\right),
$$

and

$$
\left(J_{2}^{\times} \circ d F_{1}\right)(X, 0)=J_{2}^{\times}\left(d f_{1}(X), 0\right)=\left(\varphi_{2} d f_{1}(X), \eta_{2}\left(d f_{1}(X)\right) \xi_{2}\right) .
$$

We deduce that $d f_{1} \circ \varphi_{1}=\varphi_{2} \circ d f_{1}$ and $f_{1}$ is $\varphi$-holomorphic.

Next, we take a map $f_{2}: M \rightarrow N$ from a cosymplectic manifold to a Kähler manifold. The lift $F_{2}: M^{\times} \rightarrow N:(x, y) \mapsto f_{2}(x)$ is a map between Kähler manifolds. We prove in a similar way as for $F_{1}$ :

PROPOSITION 4.5. $F_{2}$ is a holomorphic, respectively anti-holomorphic, map if and only if $f_{2}$ is $(\varphi, J)$-holomorphic, respectively $(\varphi, J)$-anti-holomorphic, that is, $d f_{2} \circ$ $\varphi=J \circ d f_{2}$, respectively $d f_{2} \circ \varphi=-J \circ d f_{2}$ (such that, in particular, $d f_{2}(\xi)=0$ ). 
Finally, starting from a map $f_{3}: N \rightarrow M$ from a Kähler manifold to a cosymplectic manifold, the lift $F_{3}: N \rightarrow M^{\times}: x \mapsto\left(f_{3}(x), a\right)$ between Kähler manifolds satisfies

PROPOSITION 4.6. $F_{3}$ is a holomorphic, respectively anti-holomorphic, map if and only if $f_{3}$ is $(J, \varphi)$-holomorphic, respectively $(J, \varphi)$-anti-holomorphic, that is, $d f_{3}$ 。 $J=\varphi \circ d f_{3}$, respectively $d f_{3} \circ J=-\varphi \circ d f_{3}$ (such that, in particular, Im $d f_{3} \perp \xi$ ).

Note that we did not mention anti-holomorphic maps in Proposition 4.4 as we did in Proposition 4.5 and Proposition 4.6, because the lift $F_{1}$ of a $\varphi$-anti-holomorphic map $f_{1}$ is anti-holomorphic only when $d f_{1}\left(\xi_{1}\right)=0$. This condition is not fulfilled for every $\varphi$-anti-holomorphic map. To see this, take an anti-holomorphic map $k: N_{1} \rightarrow N_{2}$ between Kähler manifolds and consider the map $f_{1}: N_{1} \times \mathbb{R} \rightarrow N_{2} \times \mathbb{R}:(x, t) \mapsto$ $(k(x), t)$. It is $\varphi$-anti-holomorphic for the standard cosymplectic structure on the product manifolds $N_{1} \times \mathbb{R}$ and $N_{2} \times \mathbb{R}$, and $d f\left(\xi_{1}\right)=d f(d / d t)=d / d t=\xi_{2}$.

Using these three types of lifts, we will be able to go from the level of cosymplectic manifolds to that of Kähler spaces and back again.

\section{Harmonic maps on cosymplectic manifolds}

In the theory of harmonic maps on Kähler manifolds, the following theorem is well known (see, for example, [4]).

THEOREM 5.1. If $F: N_{1} \rightarrow N_{2}$ is a holomorphic or an anti-holomorphic map between Kähler manifolds, then it is a harmonic map. If in addition $N_{1}$ is compact, then $F$ is an absolute minimum in its homotopy class for the energy functional.

We are now in a position to prove analogous results when cosymplectic manifolds are involved.

THEOREM 5.2. If $: M \rightarrow N$ is a map satisfying one of the following conditions

(a) it is a $(\varphi, J)$-holomorphic or a $(\varphi, J)$-anti-holomorphic map between a cosymplectic manifold $M$ and a Kähler manifold $N$,

(b) it is a $(J, \varphi)$-holomorphic or a $(J, \varphi)$-anti-holomorphic map between a Kähler manifold $M$ and a cosymplectic manifold $N$,

(c) it is a $\varphi$-holomorphic map between two cosymplectic manifolds, then $f$ is a harmonic map. If in addition $M$ is compact, then $f$ is an absolute minimum in its homotopy class for the energy functional.

ProOF. The proofs for the three cases are similar and follow from the results in the previous section. As an example, let us prove case (c). 
Suppose $f: M_{1} \rightarrow M_{2}$ is a $\varphi$-holomorphic map between cosymplectic manifolds. The lifted map $F: M_{1}^{\times} \rightarrow M_{2}^{\times}:(x, y) \rightarrow(f(x), f(y))$ is a holomorphic map between Kähler manifolds by Proposition 4.4, hence a harmonic map. From Proposition 4.2 it follows that also $f$ is harmonic.

Suppose now that $M_{1}$ is in addition compact. Then the same holds for $M_{1}^{\times}$and from Theorem 5.1 we know that $F$ has minimal energy within its homotopy class. Suppose that $f_{1}: M_{1} \rightarrow M_{2}$ is homotopic to $f$ via the homotopy $f_{t}$. Then the corresponding lifted mapping $F_{1}: M_{1}^{\times} \rightarrow M_{2}^{\times}$is homotopic to $F$ via the lifted homotopy $F_{t}$. So, from Proposition 4.2, $E\left(f_{1}\right)=E\left(F_{1}\right) / 2 \operatorname{vol}\left(M_{1}\right) \geq E(F) / 2 \operatorname{vol}\left(M_{1}\right)=E(f)$, and $f$ is a minimum for the energy functional within its homotopy class.

REMARK. Theorem 5.1 is also valid under the weaker conditions that $N_{1}$ and $N_{2}$ are almost Kähler. The theorem above can be strengthened accordingly to the setting where $M$ and $N$ are almost cosymplectic or almost Kähler.

For an easier formulation in the sequel, we denote from now on by a pair $(M, P)$ either a Kähler manifold, $P=J$, or a cosymplectic manifold, $P=\varphi$. A mapping $f:(M, P) \rightarrow\left(N, P^{\prime}\right)$ is $\left(P, P^{\prime}\right)$-holomorphic, respectively $\left(P, P^{\prime}\right)$-anti-holomorphic, if it satisfies $d f \circ P=P^{\prime} \circ d f$, respectively $d f \circ P=-P^{\prime} \circ d f$.

As a first corollary of Theorem 5.2, we have the following generalization of (9.21) in [3]

COROLlaRY 5.3. Let $(M, P)$ and $\left(N, P^{\prime}\right)$ be Kähler or cosymplectic manifolds with $M$ compact. If $f_{i}: M \rightarrow N$ is a smooth deformation of a $\left(P, P^{\prime}\right)$-holomorphic map through harmonic maps, then every $f_{t}$ is $\left(P, P^{\prime}\right)$-holomorphic.

As a second consequence, we have

COROLLARY 5.4. The identity map Id: $M \rightarrow M$ of a compact cosymplectic manifold is a stable harmonic map.

REMARK. This corollary can also be proved by a straightforward calculation as the one in [21] for a compact Kähler manifold.

COROLLARY 5.5. Every conformal vector field on a compact cosymplectic manifold is a Killing vector field.

PROOF. This is an immediate consequence of the previous corollary and the estimate (see, for example, [4]) index(Id) $\geq \operatorname{dim}(\mathfrak{c} / \mathrm{i}\}$, where $c$ denotes the Lie algebra of conformal vector fields and $i$ the Lie algebra of Killing vector fields.

A third application of Theorem 5.2 allows to describe a $\varphi$-holomorphic mapping between cosymplectic manifolds a little better. 
COROLLARY 5.6. Let $f: M_{1} \rightarrow M_{2}$ be a $\varphi$-holomorphic map between cosymplectic manifolds. Then $d f\left(\xi_{1}\right)=a \xi_{2}$, where $a$ is $a$ constant.

PROOF. We have noted before that $d f\left(\xi_{1}\right)=a \xi_{2}$ for some function $a$ on $M_{1}$. Then $f^{*} \eta_{2}=a \eta_{1}$ and applying the differential we find $0=d a \wedge \eta_{1}$. Hence $X a=d a(X)=$ 0 for $X \perp \xi_{1}$.

Next we use Theorem 5.2 which says that $f$ is harmonic, that is, $\tau(f)=0$. Let us compute $\tau(f)$ explicitly:

$$
\tau(f)=\alpha_{f}\left(\xi_{1}, \xi_{1}\right)+\sum_{i=1}^{n}\left(\alpha_{f}\left(e_{i}, e_{i}\right)+\alpha_{f}\left(\varphi_{1} e_{i}, \varphi_{1} e_{i}\right)\right),
$$

where $\left\{e_{1}, \ldots, e_{n}, \varphi_{1} e_{1}, \ldots, \varphi_{1} e_{n}, \xi_{1}\right\}$ is a local orthonormal frame on $M_{1}$. Using $\nabla^{M_{1}} \varphi_{1}=\nabla^{M_{2}} \varphi_{2}=0$ and the $\varphi$-holomorphicity of $f$, we calculate

$$
\begin{aligned}
\alpha_{f}\left(\varphi_{1} e_{i}, \varphi_{1} e_{i}\right) & =\nabla_{\varphi_{1} e_{i}}^{f}\left(d f\left(\varphi_{1} e_{i}\right)\right)-d f\left(\nabla_{\varphi_{1} e_{i}}^{M_{1}} \varphi_{1} e_{i}\right) \\
& =\nabla_{\varphi_{1} e_{i}}^{f}\left(\varphi_{2} d f\left(e_{i}\right)\right)-d f\left(\varphi_{1} \nabla_{\varphi_{1} e_{i}}^{M_{1}} e_{i}\right) \\
& =\varphi_{2}\left(\nabla_{\varphi_{1} e_{i}}^{f}\left(d f\left(e_{i}\right)\right)-d f\left(\nabla_{\varphi_{1} e_{i}}^{M_{1}} e_{i}\right)\right)=\varphi_{2} \alpha_{f}\left(e_{i}, \varphi e_{i}\right) \\
& =\varphi_{2}^{2} \alpha_{f}\left(e_{i}, e_{i}\right)=-\alpha_{f}\left(e_{i}, e_{i}\right)+\eta_{2}\left(\alpha_{f}\left(e_{i}, e_{i}\right)\right) \xi_{2},
\end{aligned}
$$

where we have used the symmetry of $\alpha_{f}$ in the one but last equality. The formula for $\tau(f)$ simplifies to

$$
\tau(f)=\alpha_{f}\left(\xi_{1}, \xi_{1}\right)+\sum_{i=1}^{n} \eta_{2}\left(\alpha_{f}\left(e_{i}, e_{i}\right)\right) \xi_{2} .
$$

Now $\left.\eta_{2}\left(\alpha_{f}\right)\left(e_{i}, e_{i}\right)\right)=g_{2}\left(\xi_{2}, \nabla_{e_{i}}^{f}\left(d f\left(e_{i}\right)\right)-d f\left(\nabla_{e_{i}}^{M_{1}} e_{i}\right)\right)$. On a cosymplectic manifold, $\xi^{\perp}$ is autoparallel and $d f\left(\xi_{1}^{\perp}\right) \subset \xi_{2}^{\perp}$. It follows that $\eta_{2}\left(\alpha_{f}\left(e_{i}, e_{i}\right)\right)=0$. We are left with $\tau(f)=\alpha_{f}\left(\xi_{1}, \xi_{1}\right)=\nabla_{\xi_{1}}^{f}\left(d f\left(\xi_{1}\right)\right)-d f\left(\nabla_{\xi_{1}}^{M_{1}} \xi_{1}\right)=\nabla_{\xi_{1}}^{f}\left(a \xi_{2}\right)=\xi_{1}(a) \xi_{2}$. As $f$ is harmonic, $\tau(f)=0$ and we obtain $\xi_{1}(a)=0$. So, $a$ is indeed constant.

In the context of Kähler geometry, holomorphic and anti-holomorphic mappings have been studied extensively as a special class of harmonic maps. We can now extend many of the results to situations where also cosymplectic manifolds are involved. For instance, we have the following extension of the Siu's Unique Continuation Theorem [17].

THEOREM 5.7. Let $f:(M, P) \rightarrow\left(N, P^{\prime}\right)$ be a harmonic map between manifolds $(M, P),\left(N, P^{\prime}\right)$ which are Kähler or cosymplectic. If $f$ is $\left(P, P^{\prime}\right)$-holomorphic on some open subset of $M$, then $f$ is $\left(P, P^{\prime}\right)$-holomorphic on the whole of $M$. Except for the case when $f$ is a map between two cosymplectic spaces, this also holds true for $\left(P, P^{\prime}\right)$-anti-holomorphic maps. 
PROOF. The proof is an easy application of the results of the previous section. First using the appropriate lift, we obtain a harmonic map $F$ between Kähler manifolds which is holomorphic (or anti-holomorphic) on an open subset. Applying Siu's original continuation theorem, $F$ is necessarily holomorphic (or anti-holomorphic) everywhere, hence also $f$ is $\left(P, P^{\prime}\right)$-holomorphic (or $\left(P, P^{\prime}\right)$-anti-holomorphic) everywhere on $M$.

This proof and the one of Theorem 5.2 should convince the reader that the method of making a cosymplectic manifold into a Kähler manifold and lifting mappings accordingly constitutes a very powerful tool to derive results about harmonic maps and cosymplectic spaces from analogous results in the Kähler context. We formulate two more results in this spirit to illustrate that one should be careful nonetheless.

Using the standard procedure, we can prove the following analogues of (9.12) and (9.13) from [3].

THEOREM 5.8. Let $(M, P),\left(N, P^{\prime}\right)$ be almost Kähler or almost cosymplectic with $M$ compact and such that the sectional curvature $K^{N}$ of $N$ is non-positive. Then two $\left(P, P^{\prime}\right)$-holomorphic maps which agree at a point are identical.

THEOREM 5.9. If $M$ is a compact almost cosymplectic manifold and $N$ a compact almost Kähler manifold and if the sectional curvature $K^{N}$ of $N$ is strictly negative, then there are only finitely many non-constant $(\varphi, J)$-holomorphic maps of $M$ into $N$.

The difference between these two generalizations is that the first one allows for both $M$ and $N$ to be cosymplectic whereas the second keeps a Kähler manifold as the target. The reason is that the necessary curvature condition $K^{N}<0$ is not preserved under the product construction, whereas $K^{N} \leq 0$ is.

We now simply list a few theorems on holomorphic maps on Kähler manifolds and harmonic maps involving curvature conditions, which can be easily generalized (either strongly as in Theorem 5.8 or weakly as in Theorem 5.9) to a setting involving cosymplectic manifolds. But there are probably many more.

- Theorem 3.2 in [15], a theorem due to Siu.

- Results by Yau in [23].

- (9.26) in [3], due to Lichnerowicz.

\section{6. $\varphi$-pluriharmonic maps on cosymplectic manifolds}

It is known that the composition of two harmonic maps is not necessarily a harmonic map. Harmonic morphisms are by definition mappings which pull back (local) harmonic functions to (local) harmonic functions. These maps are themselves harmonic maps which are in addition horizontally weakly conformal [7,11]. They also 
pull back (local) harmonic maps to (local) harmonic maps [12, Proposition 1]. In complex geometry, Loubeau has introduced the notion of a pluriharmonic morphism as a map pulling back (local) pluriharmonic functions to (local) pluriharmonic functions [12]. The next proposition basically says that $\varphi$-holomorphic mappings between cosymplectic manifolds qualify as ' $\varphi$-pluriharmonic morphisms'.

PROPOSITION 6.1. A smooth map $k: M_{1} \rightarrow N$ from a cosymplectic manifold $\left(M_{1}\right.$, $\left.\varphi_{1}, \eta_{1}, \xi_{1}, g_{1}\right)$ to a Riemannian manifold $N$ is $\varphi$-pluriharmonic if and only if for any $\varphi$-holomorphic map $f: M_{2} \rightarrow M_{1}$ from a cosymplectic manifold $\left(M_{2}, \varphi_{2}, \eta_{2}, \xi_{2}, g_{2}\right)$ to $M_{1}, k \circ f$ is also $\varphi$-pluriharmonic.

PROOF. First we show that the $\varphi$-holomorphic mapping $f: M_{2} \rightarrow M_{1}$ between cosymplectic manifolds is itself $\varphi$-pluriharmonic (see, for example, Proposition 3.1). As in the proof of Corollary 5.6, we easily calculate

$$
\alpha_{f}\left(\varphi_{2} X, \varphi_{2} Y\right)=\varphi_{1} \alpha_{f}\left(X, \varphi_{2} Y\right)=\varphi_{1}^{2} \alpha_{f}(X, Y)=-\alpha_{f}(X, Y)+\eta_{1}\left(\alpha_{f}(X, Y)\right) \xi_{1} .
$$

Using $d f\left(\xi_{2}\right)=a \xi_{1}$ with $a$ constant, we derive as in that same proof that $\eta_{1}\left(\alpha_{f}(X, Y)\right)=0$. Hence, $f$ is $\varphi$-pluriharmonic.

Next, we suppose that $k$ is $\varphi$-pluriharmonic and $f$ is $\varphi$-holomorphic. Then, from formula (1), we have

$$
\begin{aligned}
& \alpha_{k \circ f}(X, Y)+\alpha_{k \circ f}\left(\varphi_{2} X, \varphi_{2} Y\right) \\
&= d k\left(\alpha_{f}(X, Y)+\alpha_{f}\left(\varphi_{2} X, \varphi_{2} Y\right)\right) \\
&+\alpha_{k}(d f(X), d f(Y))+\alpha_{k}\left(\varphi_{1} d f(X), \varphi_{1} d f(Y)\right)=0 .
\end{aligned}
$$

So, $k \circ f$ is also $\varphi$-pluriharmonic.

For the converse, take for $f$ the identity mapping Id: $M \rightarrow M$.

We have similar results for $(\varphi, J)$-holomorphic and $(J, \varphi)$-holomorphic mappings, with proofs along the same lines.

PROPOSITION 6.2. Let $f: M \rightarrow N$ be $a(\varphi, J)$-holomorphic map from a cosymplectic manifold $(M, \varphi, \eta, \xi, g)$ to a Kähler manifold $(N, J, h)$. Then for any pluriharmonic map $k: N \rightarrow P$ from $N$ to a Riemannian manifold $P, k \circ f$ is $\varphi$-pluriharmonic.

Proposition 6.3. Let $f: N \rightarrow M$ be a $(J, \varphi)$-holomorphic map from a Kähler manifold $(N, J, h)$ to a cosymplectic manifold $(M, \xi, \eta, \varphi, g)$. Then for any $\varphi$ pluriharmonic map $k: M \rightarrow P$ from $M$ to a Riemannian manifold $N, k \circ f$ is pluriharmonic. 
As we have seen in the previous sections, the various forms of holomorphicity imply harmonicity when working with Kähler and cosymplectic manifolds. It is natural to ask the converse question: when does the differential of a harmonic map intertwine the structures? The problem has some answers in the Kähler case (see [15, 20]). We can now formulate analogues when the source manifold is cosymplectic.

THEOREM 6.4. Let $f: M \rightarrow N(\tilde{c})$ be a smooth map from a cosymplectic manifold $(M, \varphi, \eta, \xi, g)$ into a complex space form with constant holomorphic sectional curvature $\tilde{c} \neq 0$. Suppose that rank $d f \geq 3$ at some point of $M$. If $f$ is $\varphi$-pluriharmonic, then $f$ is $a(\varphi, J)$-holomorphic or a $(\varphi, J)$-anti-holomorphic map.

PROOF. In order to prove this result, we first lift the map $f$ to the map $F: M^{\times} \rightarrow$ $N(\tilde{c})$ as in Proposition 4.5. Now we show that $F$ is a pluriharmonic map between Kähler manifolds. We recall that $F=f \circ \pi_{1}$, where $\pi_{1}: M^{\times} \rightarrow M$ is the projection on the first factor. As $\pi_{1}$ is totally geodesic, we have for any $X=\left(X_{1}, X_{2}\right), Y=$ $\left(Y_{1}, Y_{2}\right) \in \Gamma(T(M \times M))$,

$$
\alpha_{F}(X, Y)=\alpha_{f}\left(d \pi_{1} X, d \pi_{1} Y\right) .
$$

On the other hand, it holds

$$
\begin{aligned}
\alpha_{F}\left(J^{\times} X, J^{\times} Y\right) & =\alpha_{f}\left(d \pi_{1} J^{\times} X, J^{\times} d \pi_{1} Y\right) \\
& =\alpha_{f}\left(\varphi X_{1}-\eta\left(X_{2}\right) \xi, \varphi Y_{1}-\eta\left(Y_{2}\right) \xi\right) \\
& =\alpha_{f}\left(\varphi X_{1}, \varphi Y_{1}\right)=\alpha_{f}\left(\varphi d \pi_{1} X, \varphi d \pi_{1} Y\right) .
\end{aligned}
$$

From the above two relations and the fact that $f$ is $\varphi$-pluriharmonic, we obtain

$$
\alpha_{F}(X, Y)+\alpha_{F}\left(J^{\times} X, J^{\times} Y\right)=\alpha_{f}\left(d \pi_{1} X, d \pi_{1} Y\right)+\alpha_{f}\left(\varphi d \pi_{1} X, \varphi d \pi_{1} Y\right)=0
$$

and $F$ is a pluriharmonic map from a Kähler manifold into a complex space form with non-zero holomorphic sectional curvature.

We also note that rank $d F=\operatorname{rank} d f$. So, if rank $d f \geq 3$ at some point of $M$, then by Theorem 1 in [20], we obtain that $F$ is holomorphic or anti-holomorphic. Finally, from Proposition 4.5 we get that $f$ is $(\varphi, J)$-holomorphic or $(\varphi, J)$-antiholomorphic.

REMARK. Under the same assumptions as in Theorem 6.4, if we suppose moreover that $M$ is compact, then $f$ is stable.

THEOREM 6.5. Let $f: M \rightarrow N$ be a stable $\varphi$-pluriharmonic map of a compact homogeneous cosymplectic manifold into a Kähler manifold with positive bisectional curvature. Then $f$ is a $(\varphi, J)$-holomorphic or $(\varphi, J)$-anti-holomorphic map. 
Proof. Again we lift $f: M \rightarrow N$ to the map $F: M^{\times} \rightarrow N:(x, y) \mapsto f(x)$. Then $F$ is pluriharmonic and stable (Proposition 4.3). Moreover, $M^{\times}$is still compact and homogeneous. By [15, Proposition 5.10], $F$ is holomorphic or anti-holomorphic. Proposition 4.5 finishes the proof.

As we have seen, $\varphi$-pluriharmonicity implies harmonicity. In the following proposition, we give a condition in the cosymplectic case such that harmonicity implies $\varphi$-pluriharmonicity.

PROPOSITION 6.6. Any harmonic map $f$ from a compact cosymplectic manifold to a Kähler manifold of strongly nonpositive curvature tensor is $\varphi$-pluriharmonic.

PROOF. The idea of the proof is the same as in the previous theorem. We use the lift of the map $f:(M, \varphi, \eta, \xi, g) \rightarrow(N, J, h)$ to the map $F: M^{\times} \rightarrow N$. The result follows from the corresponding result by Siu in the Kähler context (see the Introduction in [15]) and from Propositions 4.2 and 4.5.

\section{References}

[1] D. E. Blair, Contact manifolds in Riemannian geometry, Lecture Notes in Math. 509 (Springer, Berlin, 1976).

[2] M. Capursi, 'Some remarks on the product of two almost contact manifolds', An. Ştiint. Univ. Al. I. Cuza laşi Sect. I a Mat. 30 (1984), 75-79.

[3] J. Eells and L. Lemaire, 'A report on harmonic maps', Bull. London Math. Soc. 10 (1978), 1-68.

[4] - Selected topics in harmonic maps, CBMS Regional Conference Series in Mathematics 50 (American Mathematical Society, Providence, 1983).

[5] _ 'Another report on harmonic maps', Bull. London Math. Soc. 20 (1988), 385-524.

[6] J. Eells and J. H. Sampson, 'Harmonic mappings of Riemannian manifolds', Amer. J. Math. 86 (1964), 109-160.

[7] B. Fuglede, 'Harmonic morphisms between Riemannian manifolds', Ann. Inst. Fourier (Grenoble) 28 (1978), 107-144.

[8] C. Gherghe, S. Ianus and A. M. Pastore, 'CR-manifolds, harmonic maps and stability', J. Geom. $71(2001), 42-53$.

[9] A. Gray and L. M. Hervella, 'The sixteen classes of almost Hermitian manifolds and their linear invariants', Ann. Mat. Pura Appl. 123 (1980), 35-58.

[10] S. Ianus and A. M. Pastore, 'Harmonic maps on contact metric manifolds', Ann. Math. Blaise Pascal 2 (1995), 43-55.

[11] T. Ishihara, 'A mapping of Riemannian manifolds which preserves harmonic functions', $J$. Math. Kyoto Univ. 19 (1979), 215-229.

[12] E. Loubeau, 'Pluriharmonic morphisms between complex manifolds', in: Differential geometry and applications (Brno, 1998) (Masaryk Univ., Brno, 1999) pp. 89-97.

[13] E. Mazet, 'La formule de la variation seconde de l'énergie au voisinage d'une application harmonique', J. Differential Geom. 9 (1974), 531-535. 
[14] A. Morimoto, 'On normal almost contact structures', J. Math. Soc. Japan 15 (1963), 420-436.

[15] Y. Ohnita and S. Udagawa, 'Complex-analyticity of pluriharmonic maps and their constructions', in: Prospects in complex geometry (Katata and Kyoto, 1989), Lecture Notes in Math. 1468 (Springer, Berlin, 1991) pp. 371-407.

[16] A. Oubiña, 'New classes of almost contact metric structures', Publ. Math. Debrecen 32 (1985), 187-193.

[17] Y.-T. Siu, 'The complex analyticity of harmonic maps and the strong rigidity of compact Kähler manifolds', Ann. of Math. 112 (1980), 73-111.

[18] R. Smith, 'The second variation formula for harmonic mapping', Proc. Amer. Math. Soc. 47 (1975), 229-236.

[19] F. Tricerri and L. Vanhecke, 'Curvature tensors on almost Hermitian manifolds', Trans. Amer. Math. Soc. 267 (1981), 365-398.

[20] S. Udagawa, 'Pluriharmonic maps and minimal immersions of Kähler manifolds', J. London Math. Soc. 37 (1988), 375-384.

[21] H. Urakawa, Calculus of variations and harmonic maps, Transl. Math. Monographs 132 (American Mathematical Society, Providence, 1993).

[22] B. Watson, 'New examples of strictly almost Kähler manifolds', Proc. Amer. Math. Soc. 88 (1983), 541-544.

[23] S. T. Yau, 'A general Schwarz lemma for Kähler manifolds', Amer. J. Math. 100 (1978), 197-203.

Katholieke Universiteit Leuven

Department of Mathematics

Celestijnenlaan 200B

3001 Leuven

Belgium

e-mail: eric.boeckx@wis.kuleuven.ac.be
University of Bucharest Faculty of Mathematics

Str. Academiei 14

70109 Bucharest

Romania

e-mail: gherghe@gta.math.unibuc.ro 